\title{
Thermodynamics of nonsingular bouncing universes
}

\author{
Pedro C. Ferreira ${ }^{1, a}$, Diego Pavón ${ }^{2, b}$ \\ ${ }^{1}$ Escola de Ciências e Tecnologia, Universidade Federal do Rio Grande do Norte, Natal, Rio Grande do Norte 59072-970, Brazil \\ ${ }^{2}$ Departamento de Física, Universidad Autónoma de Barcelona, 08193 Bellaterra, Barcelona, Spain
}

Received: 22 September 2015 / Accepted: 5 January 2016/ Published online: 22 January 2016

(c) The Author(s) 2016. This article is published with open access at Springerlink.com

\begin{abstract}
Homogeneous and isotropic, nonsingular, bouncing world models are designed to evade the initial singularity at the beginning of the cosmic expansion. Here, we study the thermodynamics of the subset of these models governed by general relativity. Considering the entropy of matter and radiation and considering the entropy of the apparent horizon to be proportional to its area, we argue that these models do not respect the generalized second law of thermodynamics, also away from the bounce.
\end{abstract}

\section{Introduction}

The well-known, and partially successful, cosmological standard model, based on Einstein gravity, assumes a homogeneous and isotropic universe whose energy density is supplied by matter and fields that satisfy the strong energy condition [1]. The popular Einstein and de Sitter cosmology [2] is a case in point. In this class of models an inevitable singularity occurs at the beginning of the expansion. As $t \rightarrow 0$ the energy density grows beyond whatever bound, world lines cannot be continued any longer and, worse of all, the spacetime description breaks down. This holds also true if at very early times, before the conventional radiation dominated phase, the expansion of the universe is driven by some field whose energy density, essentially constant, overwhelms every other form of energy [3].

To evade this uncomfortable, philosophical, implication three main possibilities were advanced: (i) The initial singularity arises chiefly from ignoring quantum effects which are to prevail at some point before the singularity can occur [4]. (ii) The universe began expanding from a finite initial size safely larger than Planck's length (thus avoiding as well the quantum dominated regime). In this scenario, known as the "emergent" universe, an Einstein-type static phase replaces

\footnotetext{
a e-mail: pedro.ferreira@ect.ufrn.br

be-mail: diego.pavon@uab.es
}

the initial singularity - see e.g. [5,6]. (iii) Nonsingular bouncing universes. According to this proposal the universe experiences either just one contracting phase followed by an expanding one, or a sequence of expanding and contracting phases connected, in either case, to each other by a bounce such that the scale factor, $a(t)$, of the Friedmann-RobertsonWalker (FRW) metric neither vanishes nor diverges [7-12] see [13] and [14] for reviews. Since the singularity theorems of Penrose and Hawking [15] forbid bounces to originate from normal matter the physics behind them is not straightforward and requires the presence of some source of energy that violates the null energy condition. It is therefore understandable that the bounce mechanism remains a subject of debate as yet [9]. Nevertheless, nonsingular bouncing may arise in de Sitter-like models with closed spatial sections [16] as well as in supergravity-based models [17]. Figure 1 shows the evolution of the scale factor and Hubble factor, $H=\dot{a} / a$ - in arbitrary units - of a nonsingular, bouncing toy model. In spite of its simplicity, it encapsulates the salient kinematics features of this class of models.

Given the strong connection between gravity and thermodynamics [18-21], for a cosmological model that fits reasonably well the observational data it will be a bonus to comply with the laws of thermodynamics, especially the second law. The latter formalizes our daily experience that macroscopic systems, left to themselves, spontaneously tend to thermodynamic equilibrium or remain in it, if undisturbed. According to it, the entropy $S$ of isolated systems never decreases, $\dot{S} \geq 0$, and must be concave at least in the last stage of approaching equilibrium [22]. In the mid-1970s this was proved for gravity dominated systems, namely, black holes plus their environment $[23,24]$, and later extended to cosmic scenarios consisting in a de Sitter event horizon plus the fluid enclosed by it $[25,26]$.

In the case of homogeneous and isotropic universes, the entropy measured by a comoving observer splits into two parts, $S=S_{\mathcal{A}}+S_{f}$. The first one is the entropy of the 


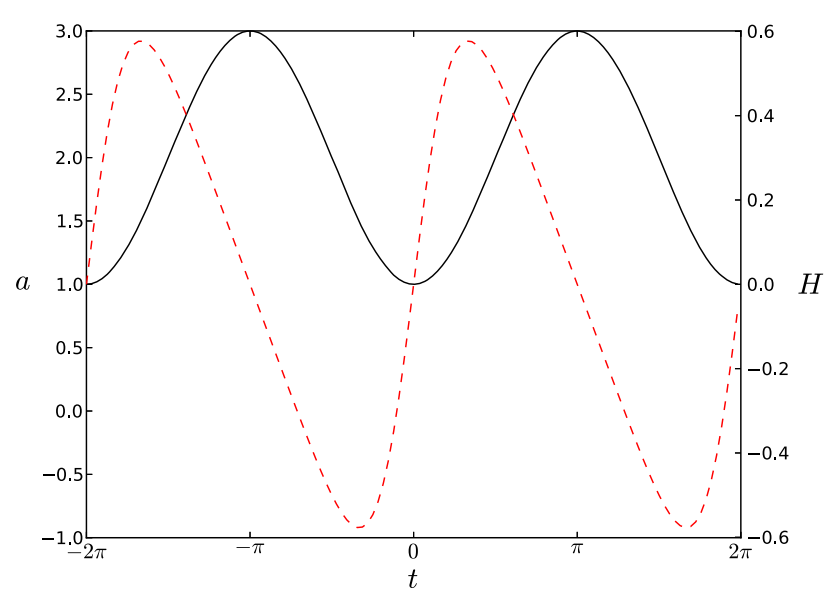

Fig. 1 Qualitative evolution of the scale factor, solid (black) line, and the Hubble function, dashed (red) line, in arbitrary units, of a nonsingular bouncing FRW universe. Here, $a=2+\sin (t)$

apparent horizon [27],

$S_{\mathcal{A}}=k_{B} \mathcal{A} /\left(4 \ell_{p l}^{2}\right)$,

where $\mathcal{A}=4 \pi \tilde{r}_{\mathcal{A}}^{2}$ denotes its area and

$\tilde{r}_{\mathcal{A}}=1 / \sqrt{H^{2}+k a^{-2}}$

denotes the horizon radius [27]. This is in keeping with the fact that the entropy of systems dominated by gravity does not vary as its volume but as the area of the surface that bounds this volume. ${ }^{1}$ The second part, $S_{f}$, corresponds to the entropy of fluids (e.g. matter and radiation) enclosed by the horizon. In many instances of interest the latter is negligible as compared with the former; this is only natural given the smallness of the $\ell_{p l}^{2}$ factor. (We do not consider the entropy of scalar fields as we assume these fields are in a pure quantum state and consequently their entropy vanishes altogether). In the particular case of the present universe the entropy of the horizon is larger than that provided by supermassive black holes, stellar black holes, relic neutrinos, and CMB photons by $18,25,33$, and 33 orders of magnitude, respectively [28].

At this point, we feel it expedient to summarize the derivation of the radius of the apparent horizon, Eq. (2). We begin by rewriting the FRW metric,

$\mathrm{d} s^{2}=-\mathrm{d} t^{2}+a^{2}(t) \frac{\mathrm{d} r^{2}}{1-k r^{2}}+a^{2}(t) r^{2} \mathrm{~d} \Omega^{2}$

(where $k=+1,0,-1$, denotes the spatial curvature index and $\Omega$ is the unit two-sphere), as

$\mathrm{d} s^{2}=h_{a b} \mathrm{~d} x^{a} \mathrm{~d} x^{b}+\tilde{r}^{2}(x) \mathrm{d} \Omega^{2}$.

1 This is, e.g., the case of the entropy of black holes
Here, $x^{0}=t, x^{1}=r$, and $h_{a b}=\operatorname{diag}\left[-1, \frac{a^{2}}{1-k r^{2}}\right]$. The radius of the dynamical apparent horizon is set by the condition $h^{a b} \partial_{a} \tilde{r} \partial_{b} \tilde{r}=0$. A straightforward calculation produces Eq. (2). To better understand the meaning of the horizon note that the expansion of the ingoing and outgoing null geodesic congruences are given by

$\theta_{\mathrm{IN}}=H-\frac{1}{\tilde{r}} \sqrt{1-\frac{k \tilde{r}^{2}}{a^{2}}}$ and $\theta_{\mathrm{OUT}}=H+\frac{1}{\tilde{r}} \sqrt{1-\frac{k \tilde{r}^{2}}{a^{2}}}$,

respectively. A spherically symmetric spacetime region will be called a "trapped" region if the expansion of ingoing and outgoing null geodesics, normal to the spatial two-sphere of radius $\tilde{r}$ centered at the origin, is negative. By contrast, the region will be called "anti-trapped" if the expansion of both kind of geodesics is positive. In normal regions outgoing null rays have positive expansion and ingoing null rays, negative expansion. Thus, the anti-trapped region is given by the condition $\tilde{r}>\left(H^{2}+k a^{-2}\right)^{-1 / 2}$. Clearly, the surface of the apparent horizon is nothing but the boundary hypersurface of the spacetime anti-trapped region. Therefore, it is only natural to associate an entropy to the apparent horizon since one can regard the latter as a measure of one's lack of knowledge about the rest of the universe. Further details concerning the entropy of the apparent horizon can be found in [27]. Obviously, in the case of an exact de Sitter expansion, the apparent and event horizons coincide.

Models falling into the emergent class fulfill the generalized second law (GSL) [29], i.e., $\dot{S}_{\mathcal{A}}+\dot{S}_{f} \geq 0$, as well as the concordance $\Lambda \mathrm{CDM}$ model [30,31]. Likewise, the models of references [32] and [33] satisfy the said law [34]. Both scenarios evade the big bang singularity and evolve from an initial de Sitter expansion to a final, also de Sitter, expansion in the far future. In the first one, [32], a gravitationally induced production of particles drives the current cosmic acceleration. In the second one, [33], the latter arises from a dynamical cosmological "constant" that depends on the Hubble factor in a manner dictated by the renormalization group [35]. By contrast, ever expanding matter-dominated universes (as the Einstein and de Sitter [2]) and phantom-dominated cosmologies obeying Einstein gravity fail to satisfy this law [30,31].

The target of this paper is to explore whether the GSL is satisfied by the nonsingular bouncing class of world models that obey general relativity, in their evolution outside the bounce itself. As mentioned above, during the bounce the null energy condition, $\rho+p \geq 0$, is violated; consequently the second law is expected to be violated as well. It is therefore worthwhile to ask whether the transgression of the second law is confined just to the bounce or also occurs in other phases of the universe evolution. This is the question we aim to answer. 
Next section focuses on a homogeneous and isotropic cosmological model, sourcered by a massive, complex, scalar field, that display a nonsingunlar bounce [7]. Section 3 studies from an overall viewpoint the class of models that incorporate matter and radiation as sources of the gravitational field though, regrettably, these models do not have analytical solutions. Section 4 presents our conclusions and final comments.

\section{Nonsingular Starobinsky's model}

Let us consider a model proposed by Starobinsky whose only energy component is a homogeneous and massive, complex, scalar field [7]. This is the simplest model featuring a nonsingular bounce that has analytical solutions for the scale factor with a nonzero measure in the space of initial conditions -in this last respect; see also [36].

The model obeys Friedmann's and energy conservation equations

$H^{2}+\frac{k}{a^{2}}=\frac{8 \pi G}{3}\left(|\dot{\varphi}|^{2}+m^{2}|\varphi|^{2}\right)$,

$\ddot{\varphi}+3 H \dot{\varphi}+m^{2} \varphi=0$

where $m$ is the quantum mass of the field and $k=+1$ in order for Eq. (6) to allow for a bounce.

At the epoch of maximum expansion $\left(-a_{\max } \ll t \ll\right.$ $\left.-m^{-1}\right)$ the universe contracts according to $a(t)=\lambda|t|^{3 / 2}$, where $\lambda$ is a positive-definite constant. In this era the variation with time of the area of the apparent horizon is

$\dot{\mathcal{A}}=-4 \pi \frac{\frac{8}{9}|t|^{-3}+\frac{4}{3} \lambda^{-2}|t|^{-7 / 3}}{\left(\frac{4}{9}|t|^{-2}+\lambda^{-2}|t|^{-4 / 3}\right)^{2}}<0$.

For $t \gg m^{-1}$ but $t<m^{-1}\left(2 \ln m a_{\max }\right)^{1 / 2}$ the scale factor obeys $a(t)=a_{1} \exp \left(-C t^{2}\right)$ with $C=m^{2} / 6$ [7]. Consequently,

$$
\dot{\mathcal{A}}=-4 \pi t \frac{8 C^{2}+\frac{4 C \exp \left(2 C t^{2}\right)}{a_{1}^{2}}}{\left(4 C^{2} t^{2}+\frac{\exp \left(2 C t^{2}\right)}{a_{1}^{2}}\right)^{2}}<0 .
$$

Figure 2 shows the evolution of the scale factor (though, in reality, the solution is valid only for a portion of the graph) as well as the evolution of the entropy of the apparent horizon.

As we see, the entropy of the horizon diminishes, at least, in some interval of the contracting stage. Since neither matter nor radiation sources the gravitational field the total entropy decreases in such periods.

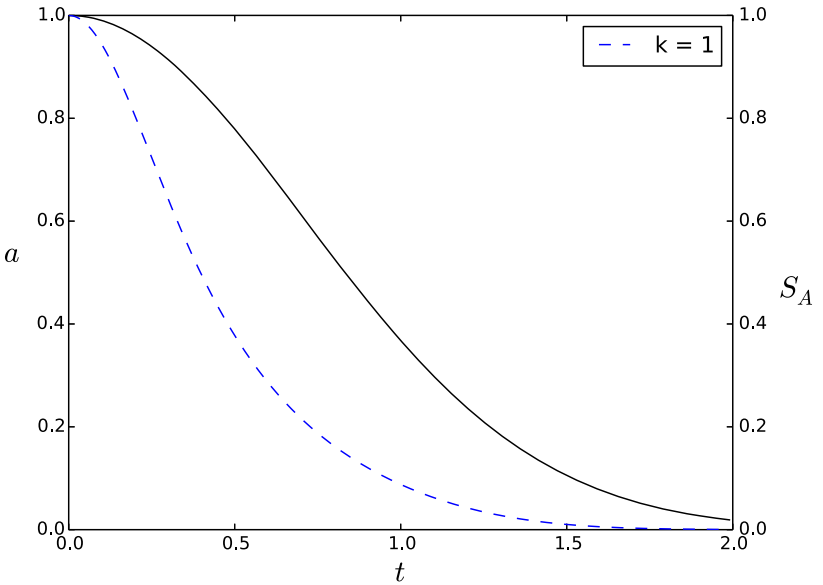

Fig. 2 Evolution of the scale factor - solid (black) line - given by $a(t)=a_{1} \exp \left(-C t^{2}\right)$, and the entropy of the horizon - dashed (blue $)$ line - for $t>0$

\section{Models with matter and radiation}

We next consider nonsingular bouncing models that include matter and radiation. Since we are not aware of analytical solutions for the scale factor our study will necessarily be from a general viewpoint. In the following we adopt the toy model of Fig. 3.

Inspection of the said figure reveals that irrespective of the sign of the curvature index, $k$, of the FRW metric (3), the entropy of the apparent horizon $S_{\mathcal{A}}$, oscillates so that it presents alternated phases of increase and decrease. To fulfill the GSL, the entropy of matter and fields inside the horizon should compensate for the stages of decrease. Even though this can hardly be the case,${ }^{2}$ this may not be necessarily true near the turning points (where $\dot{a}$ vanishes). In these points, it may depend on the details of the specific bouncing model. So, except possibly for the turning points, the entropy of matter and fields inside the horizon, most likely, will not compensate.

In Fig. 3 it is seen that when the scale factor goes from a maximum to the next minimum, the area, $\mathcal{A}=4 \pi /\left(H^{2}+\right.$ $k a^{-2}$ ) (or what amounts to the same, the entropy), of the $k=+1$ apparent horizon decreases. This is also true for the $k=0$ apparent horizon when the scale factor goes from a maximum to the consecutive inflection point. Next we shall check if matter and radiation may compensate for the decrease in entropy.

The entropy of pressureless matter, as well as of massless radiation, inside the apparent horizon varies as the number of particles in there, i.e., $S_{m}=k_{B} N_{m}, S_{\gamma}=k_{B} N_{\gamma}$ [37]. Since $N_{i}=(4 \pi / 3) n_{i} \tilde{r}_{\mathcal{A}}^{3}$, where the number densities obey $n_{m}=n_{m 0} a^{-3}$ and $n_{\gamma}=n_{\gamma 0} a^{-4}$ for matter and radiation,

2 Note that, as mentioned above, in most instances, the entropy of the horizon overwhelms any other form of entropy [28]. 


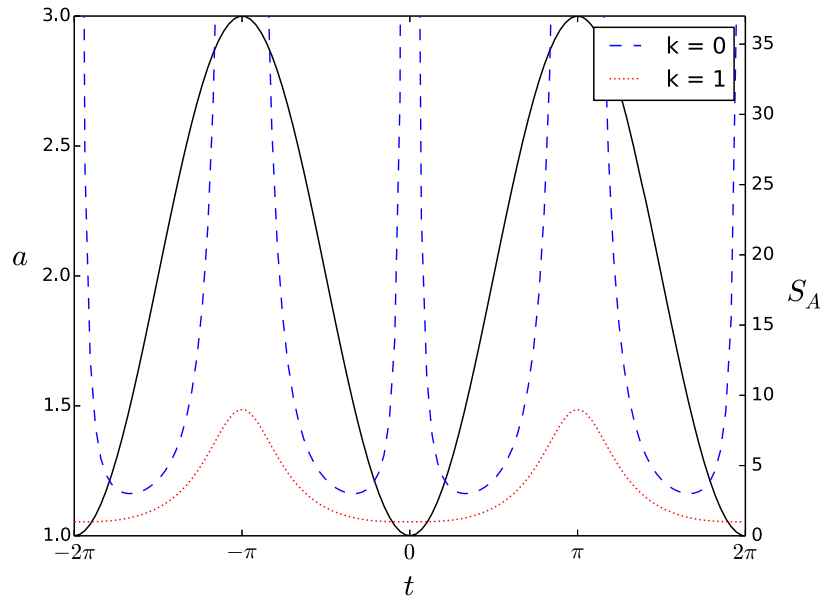

Fig. 3 Evolution of the scale factor - solid (black) line - and the horizon entropy, in arbitrary units, for $k=0-$ dashed (blue) lines, and $k=+1-$ dotted (red) line. Here, $a=2+\sin (t)$

respectively, ${ }^{3}$ one obtains

$\dot{S}_{m}=-4 \pi k_{B} n_{m 0}\left(\frac{\tilde{r}_{\mathcal{A}}}{a}\right)^{5} \dot{a} \ddot{a}$

and

$\dot{S}_{\gamma}=-4 \pi k_{B} n_{\gamma 0}\left(\frac{\tilde{r}_{\mathcal{A}}}{a}\right)^{3} H\left[\left(\frac{\tilde{r}_{\mathcal{A}}}{a}\right)^{2} \ddot{a}+\frac{1}{3 a}\right]$.

Inspection of the right-hand-side of the last two equations and Fig. 3 reveals that the entropy of the fluids (matter and radiation) enclosed by the apparent horizon also diminishes in the scale factor interval between each maximum and the subsequent inflection point (note that there both $\dot{a}$ and $\ddot{a}$ are negative quantities). Thereby, in the said interval the total entropy (that of the horizon plus that of relativistic and nonrelativistic particles inside the horizon) decreases, $\dot{S}_{\mathcal{A}}+\dot{S}_{m}+$ $\dot{S}_{\gamma}<0$.

In addition, as is well known, when matter evolves from an uniform distribution to a clumped one to form bound objects (stars, galaxies, and so on) its entropy also diminishes since spatial matter distribution goes from a disordered state to a less disordered one. In expanding phases of an oscillating universe this decrease might be counterbalanced by the entropy gain associated to the volume increase of the universe, but not in contracting phases.

Likewise, if one includes the "entropy" associated to the perturbed FRW universe, recently introduced by Clifton et al. [38], based on the Bel-Robinson tensor, the situation does not change since the said entropy is proportional to $a^{3}$ (see Eq. (54) in [38]). Then in contracting phases this entropy diminishes as well.

\footnotetext{
${ }^{3}$ For simplicity, we have set $a_{0}=1$.
}

For a bounce (or a sequence of bounces) to occur in the class of models considered in this section, an energy component that violates the null energy condition (NEC) must also be present. Thus, one may wonder if this component may restore the GSL in this context. Typically, fields that break the NEC are scalar fields of phantom type. As mentioned in the Introduction, it is generally assumed that scalar fields have vanishing entropy. Upon this usually accepted assumption, the presence of this field will not modify our results in any way. However, one may still argue that the said field might, in reality, be an effective one that corresponds to a mixture of fields, every single one being in a pure quantum state. In such a case, this effective phantom field should be entitled to an entropy. Now, the entropy of any system is an increasing function of its energy and volume. In the contracting phase, at variance with matter and fields that fulfill the NEC, the energy density of the phantom field decreases. Since the volume of the apparent horizon diminishes as well, the entropy of this effective, phantom field, decreases too. Once again, our result stands.

Altogether, our analysis suggests that the GSL is not satisfied by homogeneous and isotropic, nonsingular, bouncing models obeying Einstein relativity.

\section{Concluding remarks}

Assuming that the entropy of the universe is proportional to the area enclosing the dynamical apparent horizon, and taking into account the contributions by the matter and radiation, we investigated if nonsingular bouncing models satisfy the generalized second law of thermodynamics. We found that homogeneous and isotropic nonsingular bouncing models, governed by general relativity, do not comply with the GSL also away from the bounce. This is not only an interesting result in itself, it also begs the question as to whether more elaborated bouncing models respect this law.

One may wonder whether our results would change if we have used the event horizon rather than the apparent horizon. The answer is: no. The entropy of the former horizon is also given by his area, $\mathcal{A}_{e h} \propto l_{e h}^{2}$, where the radius of the horizon reads $l_{e h}(t)=a(t) \int_{t}^{\infty} \mathrm{d} \tilde{t} / a(\tilde{t})$. Consequently, in contracting phases this entropy decreases as well.

Similarly, the "entropy of the gravitational field" introduced four decades ago by Penrose [39,40], based on Weyl's tensor does not change our results because, in the case of nonperturbed FRW universes, the Weyl tensor vanishes identically and so does this entropy. At any rate, it faces well-known difficulties; see e.g. [41,42].

One might still argue that the failure to fulfill the GSL arises from the fact that we are observing just one universe and that we did not take into account cosmic variance. However, from the principle of indifference of Laplace [43], it is 
hard to see how cosmic variance will alter this result for the subset of the multiverse ${ }^{4}$ obeying general relativity. In any case, this depends on how one would choose a measure in the space of solutions, a subject that lies beyond the scope of this work.

Finally, bouncing models based on gravity theories that go beyond general relativity, or that introduce extra dimensions (see $[13,14]$ and references therein, as well as the recent proposals by Steinhardt et al. $[44,45]$ and Ijjas and Steinhardt [46]), should also be considered from the thermodynamic standpoint. However, for the time being, it is rather unclear how to define a meaningful entropy in such models.

We conclude by saying that, while the aforesaid connection between gravity and thermodynamics, [18-21] does not fully ensure us that the universe behaves as a thermodynamic system (i.e., one that obeys the thermodynamic laws), given two models that fit equally well the observational data, one respecting the GSL while the other does not, the former will likely be preferred.

Acknowledgments We thank the anonymous referee for useful suggestions and advice. PCF acknowledges financial support from $\mathrm{CNPq}$ (Brazil) and is grateful to the Department of Physics of the "Universidad Autónoma de Barcelona" (where part of this work was done) for warm hospitality. This research was partly supported by the "Ministerio de Economía y Competitividad, Dirección General de Investigación Científica y Técnica", Grant No. FiS2012-32099.

Open Access This article is distributed under the terms of the Creative Commons Attribution 4.0 International License (http://creativecomm ons.org/licenses/by/4.0/), which permits unrestricted use, distribution, and reproduction in any medium, provided you give appropriate credit to the original author(s) and the source, provide a link to the Creative Commons license, and indicate if changes were made. Funded by SCOAP ${ }^{3}$.

\section{References}

1. P.J.E. Peebles, Principles of Physical Cosmology (Princeton University Press, Princeton, 1993)

2. A. Einstein, W. de Sitter, Proc. Natl. Acad. Sci. 18, 214 (1932)

3. A. Linde, Particle Physics and Inflationary Cosmology (Harwood Academic, Chur, 1990)

4. Y.-F. Cai, E. Wilson-Ewing, J. Cosmol. Astropart. Phys. 03, 006 (2015)

5. G.F.R. Ellis, R. Maartens, Class. Quantum Grav. 21, 223 (2004)

6. G.F.R. Ellis, J. Murugan, C.G. Tsagas, Class. Quantum Grav. 21, $233(2004)$
7. A.A. Starobinsky, Sov. Astron. Lett. 4, 82 (1978)

8. A.Y. Kamenenshchik, I.M. Khalatnikov, A.V. Toporensky, Int. J. Mod. Phys. D 7, 673 (1997)

9. R. Brandenberger, AIP Conf. Proc. 1268, 3 (2010)

10. K. Bamba et al., J. Cosmol. Astropart. Phys. 04, 001 (2015)

11. B. Boisseau, H. Giacomini, D. Polarski, A.A. Starobinsky, J. Cosmol. Astropart. Phys. 07, 002 (2015)

12. B. Boisseau, H. Giacomini, D. Polarski, J. Cosmol. Astrpart. Phys. 10, $033(2015)$

13. M. Novello, S.E. Perez Bergliaffa, Phys. Rep. 463, 127 (2008)

14. D. Battefeld, P. Peter, Phys. Rep. 571, 1 (2015)

15. R. Penrose, S.W. Hawking, Proc. R. Soc. Lond. A314, 529 (1970)

16. J. Martin, P. Peter, Phys. Rev. D 68, 103517 (2003)

17. M. Kohen, J.L. Lehners, B.A. Ovrut, Phys. Rev. D 90, 025005 (2014)

18. J.D. Bekenstein, Phys. Rev. D 7, 2333 (1973)

19. G. Gibbons, S.W. Hawking, Phys. Rev. D 15, 2738 (1977)

20. T. Jacobson, Phys. Rev. Lett. 75, 1260 (1995)

21. T. Padmanabhan, Phys. Rep. 406, 49 (2005)

22. H. Callen, Thermodynamics (Wiley, NY, 1960)

23. J.D. Bekenstein, Phys. Rev. D 9, 3292 (1974)

24. J.D. Bekenstein, Phys. Rev. D 12, 3077 (1975)

25. P.C.W. Davies, Class. Quantum Grav. 4, L225 (1987)

26. D. Pavón, Class. Quantum Grav. 7, 487 (1990)

27. D. Bak, S.J. Rey, Class. Quantum Grav. 17, L83 (2000)

28. C.A. Egan, C.H. Lineweaver, Astrophys. J. 710, 1825 (2010)

29. S. del Campo, R. Herrera, D. Pavón, Phys. Lett. B 707, 8 (2012)

30. N. Radicella, D. Pavón, Gen. Relativ. Grav. 44, 685 (2012)

31. D. Pavón, Int. J. Geom. Methods Mod. Phys. 11, 1460007 (2014)

32. J.A.S. Lima, S. Basilakos, F.E.M. Costa, Phys. Rev. D 86, 1035034 (2012)

33. J.A.S. Lima, S. Basilakos, J. Solá, Mon. Not. R. Astron. Soc. 431, $923(2013)$

34. J.P. Mimoso, D. Pavón, Phys. Rev. D 87, 047302 (2013)

35. J. Solá, J. Phys. Conf. Ser. 283, 012033 (2011)

36. G.W. Gibbons, N. Turok, Phys. Rev. D 77, 063516 (2008)

37. S. Frautschi, Science 217, 13 (1982)

38. T. Clifton, G.F.R. Ellis, R. Tavakol, Class. Quantum Grav. 30, 125009 (2013)

39. R. Penrose, in Proceedings of the First Marcel Grossmann Meeting on General Relativity, ed. by R. Ruffini (ICTP, Triestre, North Holland, Amsterdam, 1977)

40. R. Penrose, in General Relativity: An Einstein Centenary Survey, ed. by S.W. Hawking, W. Israel (Cambridge University Press, Cambridge, 1979)

41. W.B. Bonnor, Phys. Lett. A 122, 305 (1987)

42. S.W. Goode, A.A. Coley, J. Wainwright, Class. Quantum Grav. 9, 445 (1992)

43. P.S. Laplace, in A Philosophical Essay on Probabilities, ed. by F.W. Truscott, F.L. Emory (Dover, New York, 1951)

44. P.J. Steinhardt, N. Turok, Science 296, 1436 (2002)

45. I. Bars, P. Steinhardt, N. Turok, Phys. Rev. D 89, 043515 (2014)

46. A. Ijjas, P. Steinhardt, J. Cosmol. Astropart. Phys. 10, 001 (2015)

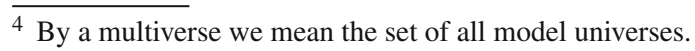

